\title{
Triplet repeat instability correlates with dinucleotide instability in primary breast cancer
}

\author{
CLAUDIA PIZZI $^{1}$, MASSIMO DI MAIO ${ }^{1}$, SANTA DANIELE ${ }^{1}$, PAOLO MASTRANZO $^{1}$, \\ ILARIA SPAGNOLETTI $^{1}$, GENNARO LIMITE ${ }^{2}$, GUIDO PETTINATO ${ }^{3}$, \\ ANTONELLA MONTICELLI ${ }^{4,5}$, SERGIO COCOZZA ${ }^{4}$ and ALMA CONTEGIACOMO ${ }^{1}$
}

\begin{abstract}
Dipartimenti: ${ }^{1}$ Endocrinologia e Oncologia Molecolare e Clinica, ${ }^{2}$ Clinico Medico-Chirurgico di Patologia dell'Apparato Digerente e di Chirurgia Generale e Oncologica, ${ }^{3}$ Scienze Biomorfologiche e Funzionali, Sezione di Anatomia Patologica,

${ }^{4}$ Biologia e Patologia Cellulare e Molecolare, Facoltà di Medicina, Università di Napoli 'Federico II'; ${ }^{5}$ IEOS, Istituto di Endocrinologia ed Oncologia Sperimentale, CNR, Via S. Pansini 5, I-80131 Napoli, Italy
\end{abstract}

Received July 25, 2006; Accepted September 1, 2006

\begin{abstract}
The expansion of triplet repeat microsatellite sequences is the molecular correlate of anticipation in a number of rare Mendelian neurodegenerative disorders. This finding prompted us to study these sequences in primary breast cancer in which there is evidence of genetic anticipation. We used a PCR/silver stain method to determine whether triplet-repeat instability (TRI) was present in DNA from malignant breast tumors, and analyzed microsatellite instability (MSI) in triplets SCA1, SCA2, SCA3, SCA6, HD, DRPLA and X25-GAA. We studied 54 consecutive primary breast cancers previously analyzed for dinucleotide instability (DI) at 9 loci. Microsatellite instability (TRI and/or DI) was found in 28/54 (52\%) cases, ranging from 0 to $56 \%$ in each patient. Dinucleotide instability occurred at $\geq 2$ loci in $19 / 54$ (35\%) cases and TRI in 6/54 (11\%). Considering single locus instability, we found DI in 26/54 (48\%) tumors and TRI in 13/54 (24\%). Triplets DRPLA and X25-GAA were most frequently unstable (14\% of cases); SCA2 instability was not detected. Interestingly, most tumors with TRI had DI (11/13, $85 \%)$. There was a correlation between TRI and DI in the same tumor (42 vs 7\% in DI+ and DI- tumors respectively, $\mathrm{p}=0.0028)$. Furthermore, TRI appears more frequently associated with lymph node metastases and more advanced clinical stages and more frequent in patients $<50$ years old, with positive steroidal hormone receptor status, positive p185 and negative p53. These findings are of interest because they demonstrate a relationship between TRI and the clinicopathological characteristics of cancer aggressiveness. Triplet
\end{abstract}

Correspondence to: Dr Alma Contegiacomo, Dipartimento di Endocrinologia e Oncologia Molecolare e Clinica, Università di Napoli 'Federico II', Via S. Pansini 5, I-80131 Napoli, Italy

E-mail: contalma@unina.it

Key words: triplet repeat instability, microsatellite instability, p53, p185, breast cancer repeat alterations can interfere with gene expression and proteomic functions, which suggests they can play a role in the neoplastic progression of mammary cells. Furthermore, the association of TRI and DI in the same tumor suggests that alterations in the DNA repair gene could culminate in selective phenotypes and breast cancer progression in a considerable number of patients.

\section{Introduction}

Eukaryotic genomes contain large numbers of repeated sequence elements of one to a few nucleotides ('microsatellites'). Interest in mutation rates in repeat sequences was stimulated by reports of associations between microsatellite instability (MSI) and human diseases and tumors $(1,2)$. Microsatellite instability is characteristic of cells lacking DNA mismatch repair, for instance, tumor cells from patients with hereditary non-polyposis colorectal cancer (3-5) and from a variety of sporadic cancers $(1,2)$. This form of instability is genome-wide and usually consists of the loss or gain of one or a few repeat units from mono-, di-, tri- and tetranucleotide microsatellites. Microsatellite instability is also associated with the hereditary neurological diseases such as Huntington disease, dominant spinocerebellar ataxy (SCA1-SCA2-SCA3 or Machado-Joseph disease-SCA6), dentatorubral-pallidolusyan atrophy (Haw-River syndrome) and Friedreich ataxia (6-13). These diseases are characterized by genes harboring tri-nucleotide repeat (TR), CNG (where $\mathrm{N}$, any nucleotide) and GAA sequences that are at risk of dynamic highfrequency mutations that expand or contract the repeat tract. Trinucleotide repeat instability (TRI) is locus-specific and can arise from gene conversion or by error-prone DNA repair whether the cell is dividing or not, since most cell types have recombination and repair properties $(14,15)$. Indeed trinucleotide repeat polymorphisms are inherently different than dinucleotide polymorphic loci. In TRI, for some repeats, large alleles above a certain threshold (i.e., expanded alleles) were shown to be pathogenic (6-14).

The discovery that expansion of triplet repeat microsatellite sequences is the molecular correlate of anticipation 
in a number of rare Mendelian neurodegenerative disorders prompted us to study these sequences in primary breast cancer in which genetic anticipation has been considered $(16,17)$. Furthermore, TRI has been reported, at various frequencies, in several sporadic tumors, i.e., colon, prostate, kidney, pancreas and testicle (18-23). Data on TRI in breast carcinomas are not extensive and mainly relate to the androgen receptor exon 1 CAG repeat length $(2,17,24-27)$. On the other side, dinucleotide repeat instability has been well characterized in primary breast cancer and correlated with histotype, tumor size, lymph node-positive phenotype, clinical stage and poorer prognosis, young age and negative p53 expression (24,28-30).

Considering that triplet repeat alterations can interfere with gene expression and proteomic functions, the aim of this study was to analyze primary breast cancer to investigate TRI in relation to dinucleotide instability (DI) and to biological and clinicopathological features in an attempt to describe new breast cancer phenotypes.

\section{Materials and methods}

Patients and tissues. Fifty-four consecutive breast-cancer patients (53 females and 1 male) were enrolled in the study. All had undergone partial or total mastectomy, with axillary lymph node clearance, and all gave their consent to the study, which was conducted in accordance with the ethical standards of the Ethics Committee of the University of Naples 'Federico II'. The age of the patients ranged between 31 and 82 years; 18 patients $(33 \%)$ were $\leq 50$, and 36 patients $(67 \%)$ $>50$ years old.

Immediately after surgery, a small portion of the tumor was rapidly frozen in liquid nitrogen and stored at $-70^{\circ} \mathrm{C}$ for DNA determination. A second specimen was placed in TC 199 tissue-culture medium (Gibco, UK) and processed for the determination of proliferative activity by the thymidine labeling index (TLI) technique (28). The remaining part was fixed in $10 \%$ buffered formalin and embedded in paraffin. Sections measuring $5 \mu$ were used for pathological and immunohistochemical studies. Only tumors with at least $50 \%$ of tumor cells were included in the study.

Tumors were classified according to histotype, grade of differentiation, tumor size, axillary lymph node status, steroid hormonal status and cell kinetics. These parameters, together with age of the patient and clinical stage of the disease, were analyzed in relation to MSI. Tumors were histologically classified according to the World Health Organization criteria (1981). The degree of differentiation of invasive ductal carcinomas was defined 'high-to-moderate' (G1/G2) or 'low' (G3), according to the Bloom and Richardson system. Steroid hormone receptor status was considered positive when the tumor expressed estrogen and/or progesterone receptor.

Thymidine labeling index. Cell kinetics was assessed by TLI technique as previously described (28). A TLI of $2.8 \%$ distinguished between tumors with low and high cell kinetics $(<2.8$ and $\geq 2.8 \%$, respectively).

Microsatellite analyses. Paired blood and tumor samples were rapidly frozen in liquid nitrogen and stored at $-70^{\circ} \mathrm{C}$. The PCR/ silver stain method we used to identify TRI in DNA from malignant breast tumors is reported elsewhere $(28,30)$. We analyzed MSI in 7 triplets: SCA1 (chr. 6), SCA2 (chr. 12), SCA3 (chr. 14), SCA6 (chr. 19), HD (chr. 4), DRPLA (chr. 12) and X25-GAA (chr. 9). The trinucleotide expanded was CAG for all genes except for X25, which was GAA. We studied 54 consecutive primary breast patients for DI at 9 loci. The dinucleotide microsatellite markers analyzed were: D1S104 (1q21-q23), D2S71 (chr. 2), D2S123 (chr. 2), D3S1611 (3p21.3), D5S107 (5q), ACTC (15q11), D17S250 (chr. 17), D17S261 (chr. 17) and D18S34 (18q21). D2S123, D5S107 and ACTC are in the reference panel recommended by the National Cancer Institute's International Guidelines for the evaluation of MSI in colorectal cancer $(2,3)$. MSI at dinucleotide and trinucleotide repeats was detected as previously reported (Fig. 1) $(13,28,30)$.

Immunohistochemical analysis. Immunohistochemistry was performed using an avidin-biotin system for mouse primary antibodies (ABC Elite Vectastain, Vector Laboratories, AL) and monoclonal antibody (MAb) DO7 directed against the p53 protein, at a dilution of 1:700 (Novocastra, UK); MAb-1 directed against $\mathrm{p} 185$, at a dilution of 1:100 (Triton, USA); 1D5 directed against estrogen receptors, at a dilution of 1:250 (Dako, DK) and 1A6 directed against progesterone receptors at a dilution of 1:250 (EPOQ, EU). Before incubation with primary antibodies, the slides were pre-treated in a microwave oven for three 5-min passages with $0.01 \mathrm{M}$ citrate buffer, $\mathrm{pH}$ 6.0. The p53 expressed in tumors was quantified and assigned to one of three categories depending on the percent of cells stained: $\leq 10 \%$, negative; $11-25 \%,(+) ; 25-50 \%,(++)$; $>50 \%,(+++)$. Two histopathologists scored slides independently and a matching hematoxylin and eosin stained slide was used to determine which part of the section was tumor tissue (30).

Statistical methods. Fisher's exact test was used for statistical analysis. P-values were computed after combining cases with an MSI phenotype defined by the presence of instability at one or more loci versus cases with no evidence of MSI. Statistical significance was considered at $\mathrm{p} \leq 0.05$.

\section{Results}

We investigated the status of microsatellites detected by random size shifts in microsatellite alleles in tumor DNA as compared with blood DNA in 54 primary breast carcinomas. Cases were collected from a consecutive series previously analyzed for DI (30). Table I shows the patients' clinicopathological characteristics. Dinucleotide instability was defined when 1 of the 9 loci analyzed was altered; TRI was defined an expansion or contraction in the length of one of the triplet repeats studied in tumor DNA with respect to blood DNA. As recommended by the National Cancer Institute guidelines for colorectal cancer $(2,3)$, the tumors were also assigned to three MSI classes: tumors with no instability (microsatellite stable); tumors with instability of at least one of the markers; tumors with instability of two or more of the markers. However, the results did not change when only tumors with more than two altered markers were considered 'positive'. 
Table I. Clinicopathological characteristics of the 54 cases analyzed.

\begin{tabular}{|c|c|c|c|c|c|c|c|c|c|c|c|c|c|}
\hline No. & Age & Histology & $\mathrm{T}$ & $\mathrm{N}$ & G & $\begin{array}{l}\text { Clinical } \\
\text { stage }\end{array}$ & TLI & $\begin{array}{l}\text { Hormonal } \\
\text { status }\end{array}$ & p185 & $\mathrm{p} 53$ & TRI & DI & MS \\
\hline 1 & 77 & CLI & $\mathrm{T} 1$ & N1 & - & III & 0.4 & + & - & - & + & + & + \\
\hline 2 & 52 & CDI & $\mathrm{T} 1$ & NO & G3 & I & 0.4 & + & - & - & - & - & - \\
\hline 3 & 65 & CDI & $\mathrm{T} 4$ & N1 & G3 & III & ND & + & + & - & - & + & + \\
\hline 4 & 76 & CLI & $\mathrm{T} 2$ & N1 & - & III & 1.1 & + & - & - & + & + & + \\
\hline 5 & 57 & CDI & $\mathrm{T} 1$ & NO & G3 & I & 0.2 & + & +++ & - & - & - & - \\
\hline 6 & 61 & CDI & $\mathrm{T} 2$ & N1 & G3 & III & 1 & - & - & ++ & - & - & - \\
\hline 7 & 72 & CDI & $\mathrm{T} 2$ & N1 & G3 & III & 4.9 & - & - & - & - & + & + \\
\hline 8 & 56 & CDI & $\mathrm{T} 2$ & No & G3 & I & 0.2 & + & - & - & - & - & - \\
\hline 9 & 70 & CDI & $\mathrm{T} 1$ & NO & G3 & I & 1.9 & - & - & +++ & - & - & - \\
\hline 10 & 54 & $\mathrm{CDI}$ & $\mathrm{T} 2$ & N1 & G3 & III & 1 & + & - & - & - & + & + \\
\hline 11 & 53 & CDI & $\mathrm{T} 2$ & N1 & G3 & III & 2.7 & + & - & - & - & - & - \\
\hline 12 & 31 & CDI & $\mathrm{T} 2$ & No & G3 & I & ND & + & - & ++ & - & - & - \\
\hline 13 & 72 & CDI & $\mathrm{T} 1$ & No & G3 & I & 0.2 & + & - & - & - & - & - \\
\hline 14 & 57 & CDI & $\mathrm{T} 1$ & No & G3 & I & 0.2 & + & +++ & + & - & - & - \\
\hline 15 & 61 & CDI & $\mathrm{T} 2$ & N1 & G3 & III & 5.1 & + & - & + & - & - & - \\
\hline 16 & 58 & CDI & $\mathrm{T} 2$ & No & G2 & I & 0.4 & + & - & - & + & + & + \\
\hline 17 & 49 & CDI & $\mathrm{T} 2$ & N1 & G3 & III & 1.2 & + & - & +++ & + & + & + \\
\hline 18 & 48 & CLI & $\mathrm{T} 2$ & N1 & - & III & 1.1 & + & - & - & - & + & + \\
\hline 19 & 51 & CDI & $\mathrm{T} 1$ & N1 & G2 & II & ND & + & - & - & - & - & - \\
\hline 20 & 41 & CDI & $\mathrm{T} 4$ & N1 & G3 & III & ND & - & - & - & - & + & + \\
\hline 21 & 48 & CLI & T3 & N1 & - & III & ND & + & - & ++ & - & - & - \\
\hline 22 & 66 & CDI & $\mathrm{T} 3$ & N1 & G3 & III & ND & - & - & - & - & + & + \\
\hline 23 & 66 & CDI & $\mathrm{T} 4$ & N1 & G3 & III & ND & - & +++ & - & + & + & + \\
\hline 24 & 76 & CLI & $\mathrm{T} 2$ & No & - & II & ND & + & - & - & - & + & + \\
\hline 25 & 61 & CDI & $\mathrm{T} 1$ & No & G3 & I & ND & - & - & - & - & - & - \\
\hline 26 & 74 & CDI & $\mathrm{T} 2$ & No & G3 & II & ND & + & +++ & +++ & - & - & - \\
\hline 27 & 72 & CLI & $\mathrm{T} 1$ & N1 & - & II & ND & + & - & - & + & + & + \\
\hline 28 & 82 & CDI & $\mathrm{T} 2$ & N1 & G3 & II & ND & + & ++ & ++ & + & - & + \\
\hline 29 & 61 & CDI & $\mathrm{T} 1$ & N1 & G3 & II & ND & + & - & - & + & - & + \\
\hline 30 & 49 & CDI & $\mathrm{T} 2$ & N1 & G3 & II & ND & ND & ND & ND & - & + & + \\
\hline 31 & 60 & CDI & $\mathrm{T} 2$ & N1 & G3 & II & ND & ND & ND & ND & - & + & + \\
\hline 32 & 59 & CDI & $\mathrm{T} 1$ & N1 & G3 & II & ND & ND & ND & ND & - & - & - \\
\hline 33 & 59 & CDI & $\mathrm{T} 1$ & No & G2 & I & ND & ND & ND & ND & - & - & - \\
\hline 34 & 78 & CDI & $\mathrm{T} 2$ & $\mathrm{Nx}$ & G1 & ND & 2.5 & + & - & + & - & - & - \\
\hline 35 & 47 & CDI & $\mathrm{T} 2$ & N1 & G3 & III & 3.5 & + & - & - & + & + & + \\
\hline 36 & 59 & CLI & $\mathrm{T} 2$ & N2 & - & III & 2.3 & - & - & - & - & - & - \\
\hline 37 & 37 & CDI & $\mathrm{T} 1$ & $\mathrm{~N} 2$ & G3 & III & 9.2 & + & - & - & - & - & - \\
\hline 38 & 65 & CDI & $\mathrm{T} 3$ & No & G3 & II & 8.4 & + & - & +++ & - & + & + \\
\hline 39 & 37 & CDI & $\mathrm{T} 1$ & N1 & G3 & III & 2.4 & + & - & - & + & + & + \\
\hline 40 & 52 & CDI & $\mathrm{T} 2$ & NO & G3 & I & 0.4 & - & - & ++ & - & - & - \\
\hline 41 & 49 & CDI & $\mathrm{T} 1$ & No & G3 & I & 2.8 & + & - & +++ & - & + & + \\
\hline 42 & 43 & CDI & $\mathrm{T} 1$ & No & G2 & I & 7.0 & + & - & - & + & + & + \\
\hline 43 & 39 & CDI & $\mathrm{T} 1$ & No & G3 & I & 6.3 & + & - & - & - & - & - \\
\hline 44 & 38 & CDI & $\mathrm{T} 2$ & N2 & G3 & III & 0.3 & - & - & - & - & + & + \\
\hline 45 & 42 & CLI & $\mathrm{T} 2$ & N1 & - & III & 0.9 & + & - & - & - & - & - \\
\hline 46 & 70 & CDI & $\mathrm{T} 2$ & No & G3 & I & 3.2 & + & - & +++ & - & + & + \\
\hline 47 & 35 & CLI & $\mathrm{Tx}$ & No & - & ND & 8.0 & + & - & - & + & + & + \\
\hline 48 & 39 & CLI & $\mathrm{T} 2$ & N1 & - & III & ND & + & - & - & - & + & + \\
\hline 49 & 48 & CLI & $\mathrm{T} 2$ & No & - & I & 0.1 & + & - & - & - & - & - \\
\hline 50 & 68 & CDI & $\mathrm{T} 1$ & No & G2 & I & 2.7 & + & - & - & - & - & - \\
\hline 51 & 74 & CLI & $\mathrm{T} 2$ & No & - & I & 2.8 & + & - & ++ & - & - & - \\
\hline 52 & 67 & CDI & $\mathrm{T} 2$ & $\mathrm{Nx}$ & G3 & ND & 4.9 & + & +++ & ++ & + & + & + \\
\hline 53 & 52 & CDI & $\mathrm{T} 1$ & No & G3 & I & 2.8 & - & - & - & - & - & - \\
\hline 54 & 39 & CDI & $\mathrm{T} 3$ & N1 & G3 & III & 4.4 & + & +++ & - & - & + & + \\
\hline
\end{tabular}

,$+ 11-25 \%$ positive cells;,$++ 26-50 \%$ positive cells;,$+++>50 \%$ positive cells. ND, not determinable. 
Table II. Triplet and dinucleotide loci status in the 54 carcinomas studied.

\begin{tabular}{|c|c|c|c|c|c|c|c|c|c|c|c|c|c|c|c|c|c|c|c|c|c|c|}
\hline No. & SCA1 & SCA2 & SCA3 & SCA6 & HD & DRPLA & $\mathrm{X} 25$ & & $\begin{array}{l}\text { RI } \\
(\%)\end{array}$ & D1S104 & $\mathrm{D} 2 \mathrm{~S} 71$ & $\mathrm{D} 2 \mathrm{~S} 123$ & D3S1611 & D5S107 & ACTC & $\mathrm{D} 17 \mathrm{~S} 250$ & D17S261 & D18S34 & & $\begin{array}{l}\text { II } \\
\text { (\%) }\end{array}$ & ${ }^{\mathrm{N}}$ & $\begin{array}{l}\text { ISI } \\
(\%)\end{array}$ \\
\hline 1 & - & - & - & + & - & - & - & 1 & 14 & - & - & - & - & + & - & - & + & + & 3 & 33 & 4 & 25 \\
\hline 2 & - & - & - & - & - & - & - & 0 & 0 & - & - & - & - & - & - & - & - & - & 0 & 0 & 0 & 0 \\
\hline 3 & - & - & - & - & - & - & - & 0 & 0 & - & - & - & - & + & - & - & - & + & 2 & 22 & 2 & 12.5 \\
\hline 4 & - & - & - & - & - & - & + & 1 & 14 & + & - & + & + & + & - & - & - & - & 4 & 44 & 5 & 31 \\
\hline 5 & - & - & - & - & - & - & - & 0 & 0 & - & - & - & - & - & - & - & - & - & 0 & 0 & 0 & 0 \\
\hline 6 & - & - & - & - & - & - & - & 0 & 0 & - & - & - & - & - & - & - & - & - & 0 & 0 & 0 & 0 \\
\hline 7 & - & - & - & - & - & - & - & 0 & 0 & + & + & - & - & - & + & - & - & - & 3 & 33 & 3 & 19 \\
\hline 8 & - & ND & - & ND & - & - & - & 0 & 0 & - & - & - & - & - & - & - & - & - & 0 & 0 & 0 & 0 \\
\hline 9 & - & - & - & - & - & - & - & 0 & 0 & - & - & - & - & - & - & - & - & - & 0 & 0 & 0 & 0 \\
\hline 10 & - & - & - & - & - & - & - & 0 & 0 & - & - & + & + & - & - & - & - & - & 2 & 22 & 2 & 12.5 \\
\hline 11 & - & - & - & - & - & - & - & 0 & 0 & - & - & - & - & - & - & - & - & - & 0 & 0 & 0 & 0 \\
\hline 12 & - & - & - & - & - & - & - & 0 & 0 & - & - & - & - & - & - & - & - & - & 0 & 0 & 0 & 0 \\
\hline 13 & - & - & - & - & - & - & - & 0 & 0 & - & - & - & - & - & - & - & - & - & 0 & 0 & 0 & 0 \\
\hline 14 & - & - & - & - & - & - & - & 0 & 0 & - & - & - & - & - & - & - & - & - & 0 & 0 & 0 & 0 \\
\hline 15 & - & - & - & - & - & - & - & 0 & 0 & - & - & - & - & - & - & - & - & - & 0 & 0 & 0 & 0 \\
\hline 16 & - & - & + & - & - & - & - & 1 & 14 & - & - & - & - & + & + & - & + & - & 3 & 33 & 4 & 25 \\
\hline 17 & + & - & + & + & + & + & + & 6 & 86 & + & - & - & + & - & - & + & - & - & 3 & 33 & 9 & 56 \\
\hline 18 & - & - & - & ND & - & - & - & 0 & 0 & - & - & + & - & - & - & - & - & + & 2 & 22 & 2 & 13 \\
\hline 19 & - & - & - & - & - & - & - & 0 & 0 & - & - & - & - & - & - & - & - & - & 0 & 0 & 0 & 0 \\
\hline 20 & - & - & - & - & - & - & - & 0 & 0 & - & - & - & - & - & + & - & - & - & 1 & 11 & 1 & 6 \\
\hline 21 & - & - & - & - & - & - & - & 0 & 0 & - & - & - & - & - & - & - & - & - & 0 & 0 & 0 & 0 \\
\hline 22 & - & - & - & - & - & - & ND & 0 & 0 & - & - & - & - & - & + & - & - & + & 2 & 22 & 2 & 13 \\
\hline 23 & - & - & - & + & - & + & + & 3 & 43 & + & - & + & + & + & + & - & - & + & 6 & 67 & 9 & 56 \\
\hline 24 & - & - & - & - & - & - & - & 0 & 0 & + & - & + & - & + & - & - & - & - & 3 & 33 & 3 & 19 \\
\hline 25 & - & - & - & - & - & - & - & 0 & 0 & - & - & - & - & - & - & - & - & - & 0 & 0 & 0 & 0 \\
\hline 26 & - & - & - & - & - & - & ND & 0 & 0 & - & - & - & - & - & - & - & - & - & 0 & 0 & 0 & 0 \\
\hline 27 & - & - & - & - & - & + & - & 1 & 14 & - & - & + & - & - & - & + & - & + & 3 & 33 & 4 & 25 \\
\hline 28 & - & - & - & - & - & + & - & 1 & 14 & - & - & - & - & - & - & - & - & - & 0 & 0 & 1 & 6 \\
\hline 29 & - & - & - & - & - & ND & + & 1 & 17 & - & - & - & - & - & - & - & - & - & 0 & 0 & 1 & 7 \\
\hline 30 & - & - & - & - & - & ND & - & 0 & 0 & - & - & - & - & - & - & + & - & + & 2 & 22 & 2 & 13 \\
\hline 31 & - & - & - & - & - & - & - & 0 & 0 & - & - & - & - & + & - & - & - & - & 1 & 11 & 1 & 6 \\
\hline 32 & - & - & - & - & - & - & - & 0 & 0 & - & - & - & - & - & - & - & - & - & 0 & 0 & 0 & 0 \\
\hline 33 & - & - & - & - & - & - & - & 0 & 0 & - & - & - & - & - & - & - & - & - & 0 & 0 & 0 & 0 \\
\hline 34 & - & - & - & - & - & - & - & 0 & 0 & - & - & - & - & - & - & - & - & - & 0 & 0 & 0 & 0 \\
\hline 35 & - & - & + & - & - & + & + & 3 & 43 & - & - & - & - & + & + & - & + & - & 3 & 33 & 6 & 37.5 \\
\hline 36 & - & - & - & - & - & - & - & 0 & 0 & - & - & - & - & - & - & - & - & - & 0 & 0 & 0 & 0 \\
\hline 37 & - & - & - & - & - & - & - & 0 & 0 & - & - & - & - & - & - & - & - & - & 0 & 0 & 0 & 0 \\
\hline 38 & - & - & - & - & - & - & - & 0 & 0 & - & - & - & - & - & - & + & - & - & 1 & 11 & 1 & 6 \\
\hline 39 & - & - & - & - & - & ND & + & 1 & 17 & + & - & - & - & - & + & + & + & - & 4 & 44 & 5 & 33 \\
\hline 40 & - & - & - & - & - & - & - & 0 & 0 & - & - & - & - & - & - & - & - & - & 0 & 0 & 0 & 0 \\
\hline 41 & - & - & - & - & - & - & - & 0 & 0 & - & - & - & - & - & - & + & - & - & 1 & 11 & 1 & 6 \\
\hline 42 & - & - & + & - & + & - & + & 3 & 43 & - & + & + & - & + & + & - & + & - & 5 & 56 & 8 & 50 \\
\hline 43 & - & - & - & - & - & - & - & 0 & 0 & - & - & - & - & - & - & - & - & - & 0 & 0 & 0 & 0 \\
\hline 44 & - & - & - & - & - & - & - & 0 & 0 & - & - & - & - & - & - & - & + & - & 1 & 11 & 1 & 6 \\
\hline 45 & - & - & - & - & - & - & - & 0 & 0 & - & - & - & - & - & - & - & - & - & 0 & 0 & 0 & 0 \\
\hline 46 & - & - & - & - & - & - & - & 0 & 0 & - & - & - & - & - & - & + & - & - & 1 & 11 & 1 & 6 \\
\hline 47 & + & - & - & - & - & + & - & 2 & 29 & + & - & + & + & - & + & - & - & - & 4 & 44 & 6 & 37.5 \\
\hline 48 & - & - & - & - & - & - & - & 0 & 0 & - & - & - & - & - & + & - & - & + & 2 & 22 & 2 & 12.5 \\
\hline 49 & - & - & - & - & - & - & - & 0 & 0 & - & - & - & - & - & - & - & - & - & 0 & 0 & 0 & 0 \\
\hline 50 & - & - & - & - & - & - & - & 0 & 0 & - & - & - & - & - & - & - & - & - & 0 & 0 & 0 & 0 \\
\hline 51 & - & - & - & - & - & - & - & 0 & 0 & - & - & - & - & - & - & - & - & - & 0 & 0 & 0 & 0 \\
\hline 52 & ND & - & - & + & - & + & - & 2 & 33 & - & - & - & - & + & - & - & + & - & 2 & 22 & 4 & 27 \\
\hline 53 & - & - & - & - & - & - & - & 0 & 0 & - & - & - & - & - & - & - & - & - & 0 & 0 & 0 & 0 \\
\hline \multirow[t]{2}{*}{54} & - & - & - & - & - & - & - & 0 & 0 & - & - & - & - & - & - & - & - & + & 1 & 11 & 1 & 6 \\
\hline & 2 & 0 & 4 & 4 & 2 & 7 & 7 & & & 7 & 2 & 8 & 5 & 10 & 10 & 7 & 7 & 9 & & & & \\
\hline$\%$ & 3.8 & 0 & 7.4 & 7.7 & 3.7 & 13.7 & 13.5 & & & 13 & 3.7 & 14.8 & 9.2 & 18.5 & 18.5 & 13 & 13 & 16.7 & & & & \\
\hline
\end{tabular}

ND, notdeterminable. 
Table III. Correlations between triplet repeats instability (TRI) and dinucleotide instability (DI) and clinicopathological characteristics in the 54 cases studied.

\begin{tabular}{|c|c|c|c|}
\hline Characteristics & No. of cases (\%) & TRI (\%) & DI (\%) \\
\hline \multicolumn{4}{|l|}{ Age } \\
\hline$\leq 50$ years & $18(33)$ & $5(28)$ & $12(77)$ \\
\hline$>50$ years & $36(67)$ & $8(22)$ & $14(39)$ \\
\hline P-value & & 0.45 & 0.05 \\
\hline \multicolumn{4}{|l|}{ Tumor size } \\
\hline$\leq 2 \mathrm{~cm}$ & $19(36)$ & $5(26)$ & $5(26)$ \\
\hline$>2 \mathrm{~cm}$ & $34(64)$ & $8(23)$ & $20(59)$ \\
\hline P-value & & 0.54 & 0.02 \\
\hline \multicolumn{4}{|c|}{ Lymph nodes status } \\
\hline $\mathrm{N}^{-}$ & $23(44)$ & $3(13)$ & $7(30)$ \\
\hline $\mathrm{N}^{+}$ & $29(56)$ & $9(31)$ & $18(62)$ \\
\hline P-value & & 0.11 & 0.02 \\
\hline \multicolumn{4}{|l|}{ Grading } \\
\hline $\mathrm{G} 1 / \mathrm{G} 2$ & $6(14)$ & $2(33)$ & $2(33)$ \\
\hline G3 & $36(86)$ & $7(20)$ & $17(47)$ \\
\hline P-value & & 0.38 & 0.43 \\
\hline \multicolumn{4}{|l|}{ Histology } \\
\hline Ductal & $42(78)$ & $5(12)$ & $19(45)$ \\
\hline Lobular & $12(22)$ & $4(33)$ & $7(58)$ \\
\hline P-value & & 0.09 & 0.32 \\
\hline \multicolumn{4}{|l|}{ Hormonal status } \\
\hline Negative & $11(22)$ & $1 \quad(9)$ & $5(46)$ \\
\hline Positive & $39(78)$ & $12(31)$ & $19(49)$ \\
\hline P-value & & 0.14 & 0.56 \\
\hline \multicolumn{4}{|l|}{ TLI } \\
\hline Low & $22(61)$ & $5(33)$ & $8(36)$ \\
\hline High & 14 (39) & $4(29)$ & $9(64)$ \\
\hline P-value & & 0.49 & 0.097 \\
\hline \multicolumn{4}{|l|}{ p185 } \\
\hline Negative & $42(84)$ & $10(24)$ & $20(48)$ \\
\hline Positive & $8(16)$ & $3(38)$ & $4(50)$ \\
\hline P-value & & 0.34 & 0.6 \\
\hline \multicolumn{4}{|l|}{ p53 } \\
\hline Negative & $34(68)$ & $10(29)$ & $19(56)$ \\
\hline Positive & $16(32)$ & 3 (19) & $5(31)$ \\
\hline P-value & & 0.33 & 0.09 \\
\hline \multicolumn{4}{|l|}{ Clinical stage } \\
\hline I & 19 (37) & $2(10)$ & $4(21)$ \\
\hline II-III & $32(63)$ & $9(18)$ & $20(67)$ \\
\hline P-value & & 0.13 & 0.004 \\
\hline
\end{tabular}

Table II shows the presence (+) and absence (-) of MSI for each dinucleotide or trinucleotide repeat, and the percentages of locus incidence and case instability. MSI (TRI and/or DI) was identified in 28 of the 54 cases $(52 \%)$. Dinucleotide instability occurred at $\geq 2$ loci in $19 / 54(35 \%)$ cases and TRI in $6 / 54(11 \%)$. With regard to single locus instability, DI occurred in 26/54 (48\%) of tumors, and TI in

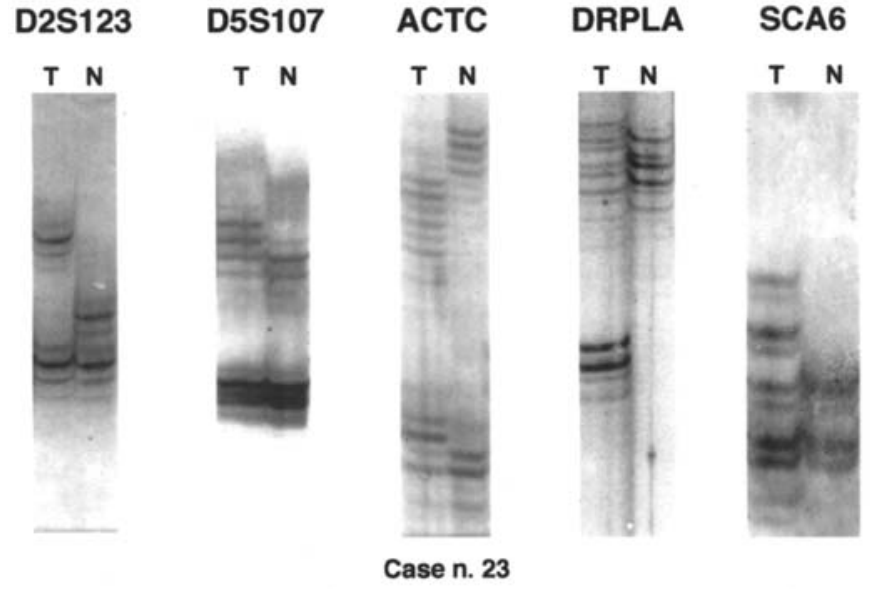

Figure 1. Examples of deranged electrophoretic mobility of PCR-fragments from tumor DNA (T) compared with normal DNA (N) (silver stain). Panel of altered loci in patient no. 23 who had high levels of DNA instability (9/16 loci, $56 \%)$.

$13 / 54(24 \%)$. The most frequently unstable triplets were DRPLA and X25-GAA (about 14\% of cases); SCA2 instability was not detected in our series. The dinucleotide loci more frequently unstable were D5S107 and ACTC (18.5\% of the cases), whereas the most stable locus was D2S71 (3.7\%). MSI ranged between 0 and $56 \%$.

Table III shows the relationships among TRI, DI and clinicopathological features. Triplet repeat instability was more frequent among patients $\leq 50$ years old, in tumors with lymphnode metastasis, in lobular histotype, in tumors with positive hormonal status, positive p185 expression, negative p53 expression and in advanced clinical stages of the disease, although correlations were not significant. Dinucleotide instability was associated with age $\leq 50$ years $(\mathrm{p}=0.05)$, tumors $>2 \mathrm{~cm}(\mathrm{p}=0.002)$, lymph node metastasis $(\mathrm{p}=0.02)$, high TLI values $(\mathrm{p}=0.097)$, negative $\mathrm{p} 53$ expression $(\mathrm{p}=0.09)$ and advanced clinical stage $(\mathrm{p}=0.004)$. Interestingly, most tumors with TRI showed DI. There was a correlation between TRI and DI in the same tumor (42 vs $7 \%$ in DI+ and DI- tumors respectively, $p=0.0028)$. In fact $11 / 13(86 \%)$ cases with TRI showed DI. Fig. 1 shows several altered loci in the patient no. 23 who had the greatest DNA instability (9/16 loci, 56\%).

\section{Discussion}

Most studies of TRI in neoplasms concern the androgen receptor and its modification in testicular germ cell cancer (22), prostate cancer (23) and breast cancer $(26,27)$. Mutations in the trinucleotide repeat ARP (arginine-rich protein) gene (3p21) have been reported in renal carcinomas (19), and in lung, breast, prostate, pancreatic and head and neck cancer (20). King et al (21) studied the trinucleotide loci SBMA, SCA1, SCA3 and DRPLA in 20 cell lines from 9 familial testicle tumors (21), and found germinal expansion of CAG repeats in 5/11 cell lines and in 1/11 sporadic testicle cancer. More recently, Giwercman et al (22) reported that the androgen receptor (AR) polymorphism was associated with both histological type and the progression rate of testicular germ cell 
cancer. In prostate carcinoma, the AR has a polyglutamine region encoded by CAG repeats in the aminoterminal domain; a reduced number of these repeats is associated with an earlier disease onset age (23).

Data on TRI in breast cancer are limited to a few studies conducted with a small number of cases $(17,24-27)$. Here, we demonstrate that in primary breast cancer TRI is a molecular marker associated to DI and earlier onset age, and not merely caused by incidental mutations. Breast cancer is characterized by genetic anticipation and more aggressive disease in younger patients $(2,16,17,26-30)$. These two features are typical of breast cancer, as they are of neurodegenerative triplet disease $(6,16,17,28-30)$.

In our series, MSI was due mostly to DI, and with a frequency similar to that of other studies $(26,28,30)$. Furthermore, loci D2S123, D5S107 and ACTC, which are recommended markers of MSI (2), are more frequently altered in primary breast cancer $(2,30)$.

All triplet repeats examined in our study were unstable in breast cancer except SCA2. But interestingly, TRI and DI were significantly associated ( $\mathrm{p}=0.0028)$. In fact, 11 of the 13 patients with TRI also had DI. This result coincides with the finding of DM-1 CAG repeat instability in 4 of 78 cases (5\%) where two of the positive cases had MSI in 9 of the 10 loci studied (17).

Our results indicate that TRI, like DI, can be considered a marker of reduced genomic stability triggered by alterations in genes involved in DNA replication and repair mechanisms $(4,5,30)$. Unstable cells will culminate in a cascade of genomic mutations that overwhelm mechanisms of cell proliferation control. TRI was identified in the E2F-4 gene, which is a transactivating factor promoting the cell cycle G1-S phase. Yoshitaka et al (18) examined 20 colorectal cancers and found alterations in the copy number of 13 consecutive trinucleotide repeats in the encoding exon E2F-4 in the two cases with MSI.

Several studies have demonstrated that MSI in primary breast cancer is predictive of recurrent and more aggressive disease (28-30). Here we confirm the relationship between DI and lymph node metastasis and larger tumor size, as well as the correlation between DI and an advanced clinical stage of the disease. DI was also more frequent in patients $\leq 50$ years old. Similar results, albeit not statistically significant, were obtained with TRI. TRI was more frequently associated with lobular carcinoma, lymph node metastases and more advanced clinical stages. It was also more frequent in patients $\leq 50$ years old, with positive steroidal hormone receptor status, positive p185 and negative p53.

In conclusion, we extend the finding of TRI, already reported in neurodegenerative disease, to primary breast cancer. We demonstrate a relationship between these mutations and the clinicopathological characteristics of cancer aggressiveness and an association with DI. Furthermore, the association of TRI and DI in the same tumor indicates that alterations in the DNA repair gene could culminate in selective breast cancer progression in a considerable number of patients. Further studies need to verify whether TRI respect to DI (or MSI) correlates to outcome or response to treatment.

\section{Acknowledgements}

The study was supported by MURST COFIN 2003.

\section{References}

1. Arzimanoglou II, Gilbert F and Barber HR: Microsatellite instability in human solid tumors. Cancer 82: 1808-1820, 1998.

2. Boland CR, Thibodeau SN, Hamilton SR, Sidransky D, Eshleman JR, Burt RW, Meltzer SJ, Rodriguez-Bigas MA, Fodde R, Ranzani GN and Srivastava S: A National Cancer Institute workshop on microsatellite instability for cancer detection and familial predisposition: development of international criteria for determination of microsatellite instability in colorectal cancer. Cancer Res 58: 5248-5257, 1998.

3. Pinol V, Castells A, Andreu M, Castellvi-Bel S, Alenda C, Llor X, Xicola RM, Rodriguez-Moranta F, Paya A, Jover R and Bessa X: Gastrointestinal Oncology Group of the Spanish Gastroenterological Association: accuracy of revised Bethesda guidelines, microsatellite instability, and immunohistochemistry for the identification of patients with hereditary non-polyposis colorectal cancer. JAMA 293: 1986-1994, 2005.

4. Liu B, Parsons R, Papadopoulos N, Nicolaides NC, Lynch HT, Watson P, Jass JR, Dunlop M, Wyllie A, Peltomaki P, De la Chapelle A, Hamilton SR, Vogelstein B and Kinzler KW: Analysis of mismatch repair genes in hereditary non-polyposis colorectal cancer patients. Nature Med 2: 169-174, 1996.

5. Oda S, Maehara Y, Ikeda Y, Oki E, Egashira A, Okamura Y, Takahashi I, Kakeji Y, Sumiyoshi Y, Miyashita K, Yamada Y, Zhao Y, Hattori H, Taguchi K, Ikeuchi T, Tsuzuki T, Sekiguchi M, Karran P and Yoshida MA: Two models of microsatellite instability in human cancer: differential connection of defective DNA mismatch repair to dinucleotide repeat instability. Nucleic Acids Res 33: 1628-1636, 2005.

6. Wilmot GR and Warren ST: Genetic Instabilities and Hereditary Neurological Diseases. Wells RD and Warren ST (eds). Academic Press, San Diego, CA, pp3-12, 1998.

7. The Huntington's Disease Collaborative Research Group: A novel gene containing a trinucleotide repeat that is expanded and unstable on Huntington's disease chromosomes. Cell 72: 971-983, 1993.

8. Orr HT, Chung M, Banfi S, Kwiatkowski JR, Servadio A, Beaudet AL, McCall AE, Duvick LA, Ranum LPW and Zoghbi HY: Expansion of an unstable trinucleotide (CAG) repeat in spinocerebellar ataxia type 1. Nat Genet 4: 221-226, 1993.

9. Imbert G, Saudou F, Yvert G, Devys D, Trottier Y, Garnier JM, Weber C, Mandel JL, Cancel G, Abbas N, Durr A, Didierjean O, Stevanin G, Agid Y and Brice A: Cloning of the gene for spinocerebellar ataxia 2 reveals a locus with high sensitivity to expanded CAG/glutamine repeats. Nat Genet 14: 2285-2291, 1996.

10. Klockgether T, Kramer B, Ludtke R, Schols L and Laccone F: Repeat length and disease progression in spinocerebellar ataxia 3. Lancet 348: 830, 1996.

11. Zhuchenko O, Bailey J, Bonnen P, Ashizawa T, Stockton DW, Amos C, Dobyns WB, Subramony SH, Zoghbi HY and Lee CC: Autosomal dominant cerebellar ataxia (SCA6) associated with small polyglutamine expansions in the $\alpha 1 \mathrm{~A}$-voltage-dependent calcium channel. Nat Genet 15: 62-69, 1997.

12. Burke J, Ikeuchi T, Koide R, Tsuji S, Yamada M, PericakVance $\mathrm{M}$ and Vance J: Dentatorubral-pallidolusyan atrophy and Haw River syndrome. Lancet 344: 1711-1712, 1994.

13. Campuzano V, Montermini L, Molto PD, Pianese L, Cossee M, Cavalcanti F, Monros E, Rodius F, Duclos F, Monticelli A, Zara F, Canizares J, Koutnikova H, Bidichandani SI, Gellera C, Brice A, Trouillas P, De Michele G, Filla A, De Frutos R, Palau F, Patel PI, Di Donato S, Mandel JL, Cocozza S, Koenig M and Pandolfo M: Friedreich's ataxia: autosomal recessive disease caused by an intronic GAA triplet repeat expansion. Science 271: 1374-1375, 1996.

14. Caskey T, Pizzuti A, Ying-Fui F, Raymond G, Fenwick JR and Nelson D: Triplet repeat mutations in human disease. Science 256: 784-789, 1992.

15. Loeb LA: Mutator phenotype may be required for multistage carcinogenesis. Cancer Res 51: 3075-3079, 1991.

16. Paterson AD, Naimark DM, Huang J, Vachon C, Petronis A, King RA, Anderson VE and Sellers TA: Genetic anticipation and breast cancer: a prospective follow-up study. Breast Cancer Res Treat 55: 21-28, 1999. 
17. Shaw JA, Walsh T, Chappell SA, Carey N, Johnson K and Walker RA: Microsatellite instability in early sporadic breast cancer. Br J Cancer 73: 1393-1397, 1996.

18. Yoshitaka T, Matsubara N, Ikeda M, Tanino M, Hanafusa H, Tanaka N and Shimizu K: Mutations of E2F-4 trinucleotide repeats in colorectal cancer with microsatellite instability. Biochem Biophys Res Commun 227: 553-557, 1996.

19. Shridhar R, Shridhar V, Rivard S, Siegfried JM, Pietraszkiewicz H, Ensley J, Pauley R, Grignon D, Sakr W, Miller OJ and Smith DI: Mutations in the arginine-rich protein gene, in lung, breast and prostate cancers, and in squamous cell carcinoma of the head and neck. Cancer Res 56: 5576-5578, 1996.

20. Shridhar V, Rivard S, Wang X, Shridhar R, Paisley C, Mullins C, Beirnat L, Dugan M, Sarkar F, Miller OJ, Vaitkevicius VK and Smith DI: Mutations in the arginine-rich protein gene (ARP) in pancreatic cancer. Oncogene 14: 2213-2216, 1997.

21. King BL, Peng H-Q, Goss P, Huan S, Bronson D, Kacinski BM and Hogg D: Repeat expansion detection analysis of (CAG)n tracts in tumors cell lines, testicular tumors and testicular cancer families. Cancer Res 57: 209-214, 1997.

22. Giwercman A, Lundin KB, Eberhard J, Stahl O, Cwikiel M, Cavalli E and Giwercman YL: Linkage between androgen receptor gene CAG Trinucleotide repeat length and testicular germ cell cancer histological type and clinical stage. Eur J Cancer 40: 2152-2158, 2004.

23. Stanford JL, Just JJ, Gibbs M, Wicklund KG, Neal CL, Blumenstein BA and Ostrander EA: Polymorphic repeats in the androgen receptor gene: molecular markers of prostate cancer risk. Cancer Res 57: 1194-1198, 1997.
24. Ottini L, Palli D, Falchetti M, D'Amico C, Noviello C, Saieva C, Russo A, Corsi AM, Masala G, Paglierani M, Vezzosi V, Bianchi S and Mariani-Costantini R: Instability at dinucleotide and trinucleotide repeats in breast cancer. Int J Oncol 17: 819-826, 2000.

25. Day P and Wong LJ: Somatic instability of the DNA sequences encoding the polymorphic polyglutamine tract of the AIB 1 gene. J Med Genet 40: 885-890, 2003.

26. Yu H, Bharaj B, Vassilikos EJ, Giai M and Diamandis EP: Shorter CAG repeat length in the androgen receptor gene is associated with more aggressive forms of breast cancer. Breast Cancer Res Treat 59: 153-161, 2000.

27. Elhaji YA, Gottlieb B, Lumbroso R, Beitel LK, Foulkes WD, Pinsky L and Trifiro MA: The polymorphic CAG repeat of the androgen receptor gene: a potential role in the breast cancer women over 40. Breast Cancer Res Treat 70: 109-116, 2001

28. De Marchis L, Contegiacomo A, D'Amico C, Palmirotta R, Pizzi C, Ottini L, Mastranzo P, Figliolini M, Petrella G, Amanti C, Battista P, Bianco AR, Frati L, Cama A and Mariani-Costantini R: Microsatellite instability is correlated with lymph node-positive breast cancer. Clin Cancer Res 3: 241-248, 1997.

29. Paulson TG, Wright FA, Parker BA, Russack V and Wahl GM: Microsatellite instability correlates with reduced survival and poor disease prognosis in breast cancer. Cancer Res 56: 4021-4026, 1996.

30. Pizzi C, Panico L, De Marchis L, Mastranzo P, Di Maio M, D'Amico C, Limite G, Pettinato G, Cocozza S, Bianco AR and Contegiacomo A: p53 expression is decreased in primary breast carcinomas with microsatellite instability. Breast Cancer Res Treat 73: 257-266, 2002. 